\title{
Degradation of a Dielectric Barrier Discharge Plasma Actuator
}

\author{
Andrew Ragai Henry RIGIT*, Koon Chun LAI, David Boon Liang BONG \\ Faculty of Engineering, Universiti Malaysia Sarawak, 94300 Kota Samarahan, Malaysia \\ *E-mail: arigit@feng.unimas.my
}

\begin{abstract}
Dielectric barrier discharge is vulnerable to ion bombardment, radical species or ultraviolet radiations that can be emitted by plasma filaments in air under atmospheric pressure. In our experiments, traces of degradation on the actuator surface can be observed by naked eye after the discharge operation. The degradation could come from the non-uniformity of the electric field. Despite the degradation marks, some scratches due to the corona discharge process can be seen on the dielectric surface. The parametric study in this study reveals that the degradation on the actuator panel is subjected to a failure rate that increases with the cumulative time of plasma operation and the magnitude of supplied voltage. Besides, this study suggests that the severity of degradation can be lessened for a symmetric and larger gap design plasma actuator, since the concentration for the ion bombardment can be weakened at a particular discharge area.
\end{abstract}

Keywords: Plasma actuator, corona discharge, dielectric degradation

\section{INTRODUCTION}

Since decades, plasma science is a field of growing interest. Plasma, which defines the ionized state of gases, has become increasingly important for various industrial applications [1]. The device in-used, plasma actuator, is named after its ability to establish the electric wind or ionic flow. There are two most-used actuators, namely corona discharge plasma actuator and dielectric barrier discharge (DBD) plasma actuator [2]. The latter design will be analysed in this study.

Dielectric barrier discharge (DBD), which also known as surface DBD (SDBD) has been widely used for the industrial applications and aerodynamic flow control $[3,4]$. It is a nonthermal discharge under atmospheric pressure that can generate UV emission and low-temperature plasma in the air. The discharge is performed at the surface of a dielectric layer between several electrodes mounted flush at both sides in an asymmetric configuration. A schematic diagram of the experimental layout and essential connections is demonstrated by Fig. 1.

The dielectric barrier can be made of glass, quartz, ceramics or polymer materials of low dielectric loss and high breakdown strength. The DBD proceeds in most gases or atmospheric air through a large number of separate current filaments referred to as microdischarges. These microdischarges have complex dynamic structure and are formed by channel streamers that repeatedly strike at the same place as the polarity of the applied voltage changes, appearing to the eye as bright filaments [5]. Charge accumulated on the surface of the dielectric barrier reduces the electric field at the location of a microdischarge, resulting in current termination within tens of nanoseconds after breakdown. The short duration of current in microdischarges leads to low heat dissipation, causing the DBD plasma to remain strongly non-thermal.

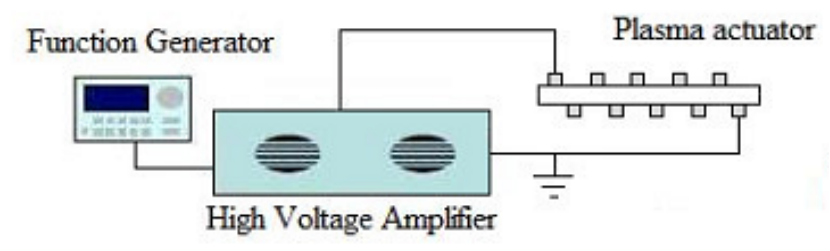

Fig. 1. Experimental Setup

Dielectrics in contact with plasma processes are subjected to electrical charging. The establishment of the electric charge distribution in dielectrics can have a profound influence on the degradation and roughness of the materials. The roughness and cavities caused by the degradation should be prevented since lower roughness can result in better electrical properties, such as high mobility, low leakage current and better reliability [6]. Recently, it had been reported in their papers that the degradation of dielectric can be observed on the DBD plasma actuator panel, after being exposed to the plasma generation at atmospheric pressure $[7,8]$. Owed to the facts that the lower surface roughness can result in better electrical properties, a parametric study was carried out to facilitate further understandings in relation to the severity level of the dielectric degradation. Three relevant parameters, namely, operation duration, magnitude of supplied voltage and electrode geometry, were evaluated on their impacts to the degradation process.

\section{PLASMA ACTUATOR PANEL DESIGN}

The plasma actuator panel under tested consists of two identical copper actuators (top and bottom) with an intermediate dielectric layer, as shown in Fig. 2, built under standard printed circuit board (PCB) fabrication process. The dielectric layer, made of PCB materials, 